\title{
Gd disilicide nanowires attached to Si(111) steps
}

\author{
J. L. McChesney, A. Kirakosian, R. Bennewitz ${ }^{\text {, }}$ J. N. Crain, J.-L. Lin, and F. J. Himpsel \\ Department of Physics, UW-Madison, 1150 University Ave., Madison, WI 53706, USA \\ ${ }^{a}$ Dept. of Physics and Astronomy, University of Basel, 4056 Basel, Switzerland
}

\begin{abstract}
Self-assembled electronic devices, such as quantum dots or switchable molecules, need self-assembled nanowires as connections. We explore the growth of conducting Gd disilicide nanowires at step arrays on $\mathrm{Si}(111)$. Atomically smooth wires with large aspect ratios are formed at low coverage and high growth rate (length $>1 \mu \mathrm{m}$, width $10 \mathrm{~nm}$, height $0.6 \mathrm{~nm}$ ). They grow parallel to the steps in the [ $\left.\begin{array}{lll}1 & \overline{1} & 0\end{array}\right]$ direction, which is consistent with a lattice match of $0.8 \%$ with the a-axis of the hexagonal silicide, together with a large mismatch in all other directions. This mechanism is similar to that observed previously on $\mathrm{Si}(100)$. In contrast to $\mathrm{Si}(100)$, the wires are always attached to step edges on $\mathrm{Si}(111)$ and can thus be grown selectively on regular step arrays.
\end{abstract}

With the advent of molecular electronics using self-assembled nanodots and nanotubes as electronic components there is a growing need for self-assembled interconnects. So far, electron beam lithography has been used for wiring individual devices, such as a memory cell or a transistor. This technique is not viable for assembling such devices by the billions. Therefore, a variety of methods have been studied for the directed self-assembly of wires between electrodes, for example selective bonding of a long DNA strand to short, complementary pieces of DNA at the electrodes and subsequent metallization [1].

Silicon surfaces are the traditional substrate for electronic devices, which makes them prime candidates for growing nanowires. It was found early on that silver nanowires with high aspect ratio can be obtained at $\mathrm{Si}(100)$ surfaces [2]. The explanation for such anisotropic growth behavior was a minimization of the strain energy by relaxing the strain perpendicular to a narrow wire while keeping it fully-strained in the long direction. More recently, transition metals [3-6] and rare earth [711] disilicides have attracted attention because of their anisotropic growth mode on $\mathrm{Si}(100)$. In that case the strain is anisotropic to begin with, due to good lattice match parallel to the growth direction combined with a mismatch perpendicular to it. For example, the hexagonal, Si-deficient $\mathrm{GdSi}_{1.65}$ phase has a lattice match of $0.8 \%$ between its a-axis and the $\left[\begin{array}{llll}1 & \overline{1} & 0\end{array}\right]$ direction of $\mathrm{Si}$ while being mismatched by $10-20 \%$ in other orientations $[12,13]$. Since $\left[\begin{array}{lll}1 & \overline{1} & 0\end{array}\right]$ is an in-plane direction common to both $\mathrm{Si}(100)$ and $\mathrm{Si}(111)$, there is the possibility of growing nanowires on both surfaces. Further benefits of these silicides are their conductivity (e.g., $190 \mu \Omega \mathrm{cm}$ for hexagonal $\mathrm{GdSi}_{1.65}$ at room temperature [13]) and their extremely low Schottky barrier to n-type silicon [14]. Another feature of interest is their magnetism (anitferromagnetic below $\mathrm{T}_{\mathrm{N}}=33 \mathrm{~K}$ ).

A primary requirement for the use of nanowires in self-assembled devices is the ability to control their location. If the active electronic elements, such as memory cells and switches, are assembled first, then the wiring has to find their location and start the growth there. Alternatively, a wire matrix can be assembled first, and then the switching components inserted at the cross-links $[15,16]$. Previous work with rare earth disilicide nanowires has focused on the $\mathrm{Si}(100)$ orientation, where two sets of orthogonal nanowires grow at the two types of terraces that are rotated by $90^{\circ}$. However, the wires apparently nucleate at random locations on the surface [7-11]. Therefore, we have turned our attention to $\mathrm{Si}(111)$, where it is possible to produce highly-regular arrays of steps [17] that might serve as templates for forming wire arrays. Indeed, we find that Gd disilicide wires exclusively grow along step edges on $\mathrm{Si}(111)$, as shown in Figs. 1 and 2. That suggests their possible use in crossedwire arrays with back-to-back wafers bonded by the molecules or nanodots.

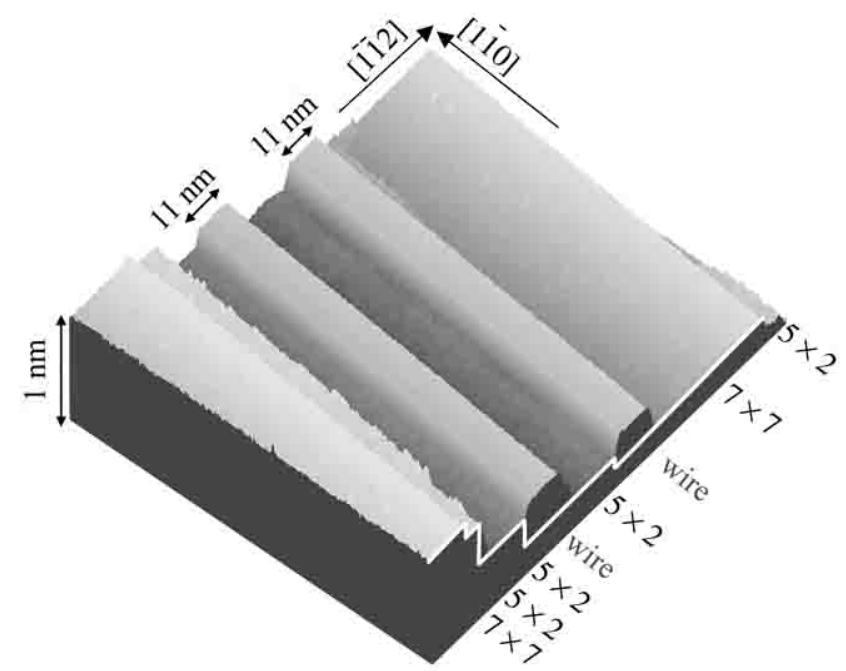

Fig. 1 Two Gd disilicide nanowires attached to step edges of $\mathrm{Si}(111)$. Note the atomically-precise widths of $11 \mathrm{~nm}$. (STM image at $+1.2 \mathrm{~V}$ sample bias). 


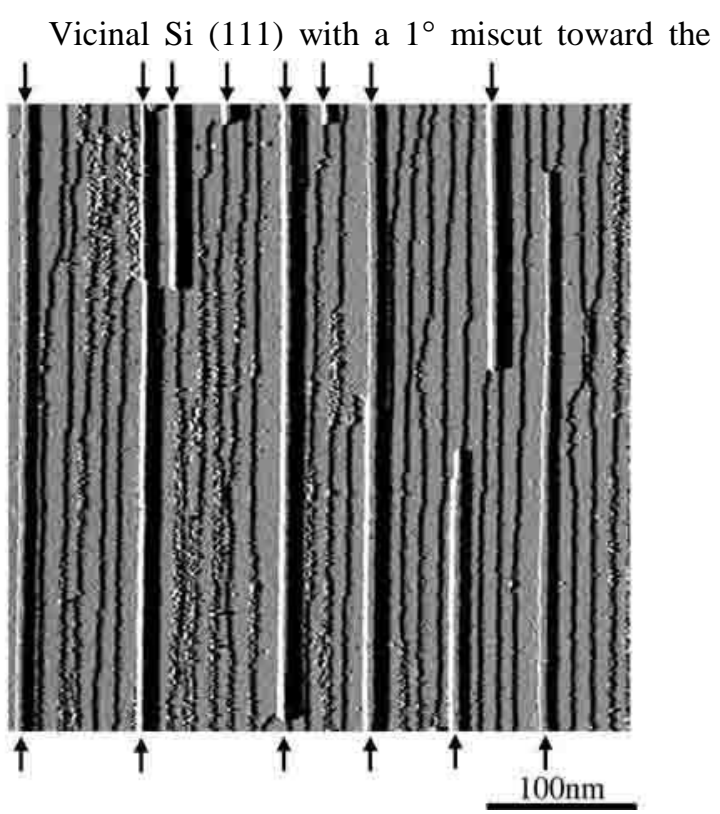

Fig.2 STM image of several Gd disilicide nanowires (arrows) that are all attached to step edges of Si(111) running along the [ $\left.\begin{array}{lll}1 & \overline{1} & 0\end{array}\right]$ direction. The $\mathrm{x}$-derivative of the topography is shown in this and the following images. Wires appear to be illuminated from the left and casting shadows to the right, which is downhill.

[ $\left.\begin{array}{lll}\overline{1} & \overline{1} & 2\end{array}\right]$ azimuth serves as a template since the $\left[\begin{array}{lll}1 & \overline{1} & 0\end{array}\right]$ step direction has an $0.8 \%$ lattice match with the a-axis of bulk hexagonal silicide. Perpendicular to the step (along the [ $\left.\begin{array}{lll}\overline{1} & \overline{1} & 2\end{array}\right]$ direction) there is a large mismatch, no matter which orientation the silicide takes relative to the $\mathrm{Si}$ substrate. If the c-axis lies perpendicular to the surface, as reported in [12] for silicide films, the mismatch is $9 \%$. If the c-axis is in-plane the mismatch is $28 \%$. For optimal growth of long wires distributed evenly across the surface, 0.05 monolayers of $\mathrm{Gd}$ where deposited at a rate of 1.3 $\AA / m i n$ while keeping the sample at $675^{\circ} \mathrm{C}$. Although other temperatures and flux rates produced wires, these parameters produced the longest and most densely spaced wires.

There is a very limited window within which wires will be produced due to the kinetic nature of the process. The flux rate needs to exceed the diffusion rate of $\mathrm{Gd}$ into bulk $\mathrm{Si}$, which is significant at $700^{\circ} \mathrm{C}$ [18]. Furthermore, the Gdinduced $\mathrm{Si}(111) 5 \times 2-\mathrm{Gd}$ surface structure [19] competes with nanowires for available Gd. Though $\mathrm{Si}(111) 5 \times 2-G d$ can coexist with nanowires on the same surface (see Fig. 1), the highest wire densities were observed when the remaining surface was still clean $\operatorname{Si}(111) 7 \times 7$. There is a second Gd-induced structure at higher coverage than $5 \times 2-\mathrm{Gd}$, i.e. $\operatorname{Si}(111) \sqrt{3} \times \sqrt{3}-$ Gd. It is able to coexist with nanowires as well.

Typical dimensions of the nanowires are a length of $>1 \mu \mathrm{m}$, a width of $10 \mathrm{~nm}$, and a height of $0.6 \mathrm{~nm}$. Figure 3 shows wires in the $\mu \mathrm{m}$ range, whose length is mainly limited by kinks of the underlying $\mathrm{Si}(111)$ steps. In fact, the wires straighten out the steps to atomic smoothness until a step deviates so far from a straight line that it requires too much $\mathrm{Si}$ needs to be displaced to straighten out the step. This is reminiscent of the growth mode on the $\mathrm{Si}(100)$ surface, where a nanowire can grow only on the same terrace. It extends an atomically flat terrace as far as feasible within the limited Si diffusion rate by building up material on a downhill terrace or burrowing into an uphill terrace. Such an effect is also seen on $\mathrm{Si}(111)$.

The width of the nanowires remains atomically precise along the wire in most cases (see Figs. 1-3). The most frequent width is $11 \mathrm{~nm}$ (Fig. 1), but widths between 5 and $20 \mathrm{~nm}$ have been observed. Occasional changes in the wire width tend to occur in multiples of $0.4 \mathrm{~nm}$, which would indicate that this is the maximum width for dislocation-free epitaxy perpendicular to the wires.

Strain confines not only the wire width, but the height as well. All wires are $0.6 \mathrm{~nm}$ high relative to the bottom terrace, independent of wire density or deposition time. Only slight variations are seen $(0.05 \mathrm{~nm})$, which can be traced to different substrate reconstructions and tips with differences in the electronic structure.

The Gd disilicide nanowires are rather inert and stable. After being exposed to residual gas at

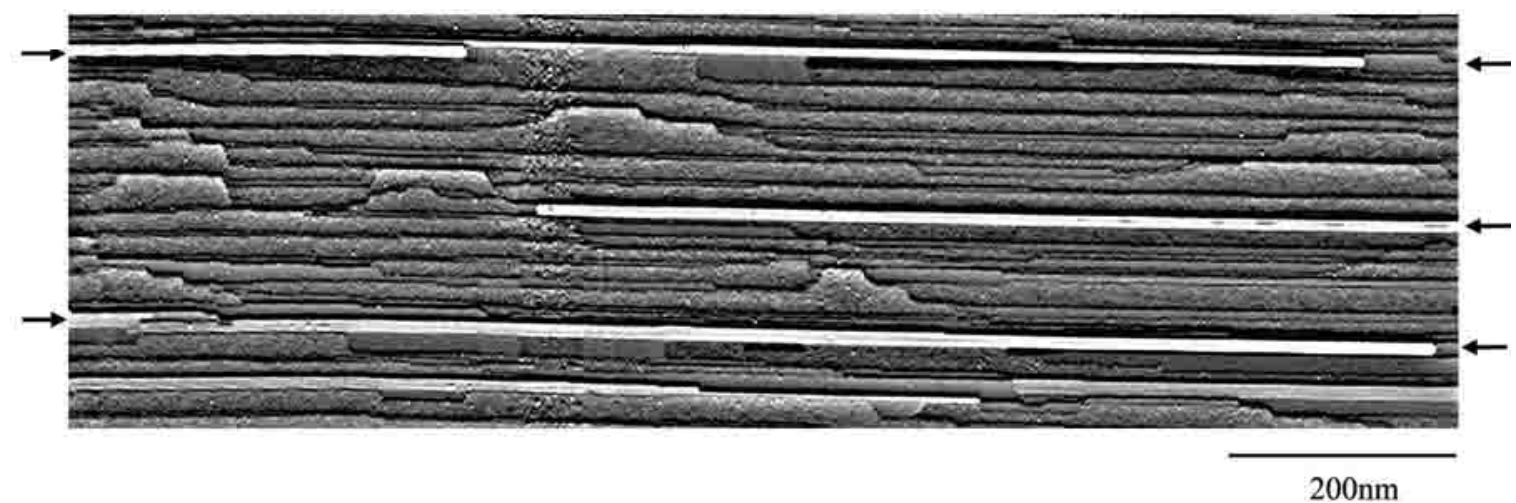

Fig. 3 Nanowires reaching $\mu \mathrm{m}$ dimensions. Their length is limited by the straightness of the Si steps, because the wires do not form kinks. 
$1 \times 10^{-10}$ Torr for several days, the wires are unaffected while the $5 \times 2-\mathrm{Gd}$ structure becomes completely covered with adsorbates (grainy patches in Fig. 2). After several $10 \mathrm{~min}$ anneals at $700^{\circ} \mathrm{C}$ the wires appear unchanged but the surrounding $5 \times 2$-Gd surface converts back to a mixture of clean $7 \times 7$ and patches of multi-domain $5 \times 2-\mathrm{Gd}$.

The surface of the wires exhibits chain like surface reconstructions, one with a chain spacing of two silicon surface lattice constants, the other with four. Both are shown Fig. 4, where a nanowire is sandwiched between a $5 \times 2-G d$ terrace on the left and a $7 \times 7$ terrace on the right. There is a significant change when tunneling from occupied orbitals ( $-2 \mathrm{~V}$ sample bias) to tunneling into unoccupied orbitals $(+2 \mathrm{~V}$ bias), which hints at a pronounced modulation of the charge distribution perpendicular to the chains, similar to that seen for the $5 \times 2-\mathrm{Gd}$ structure [19].

In summary, we have observed spontaneous

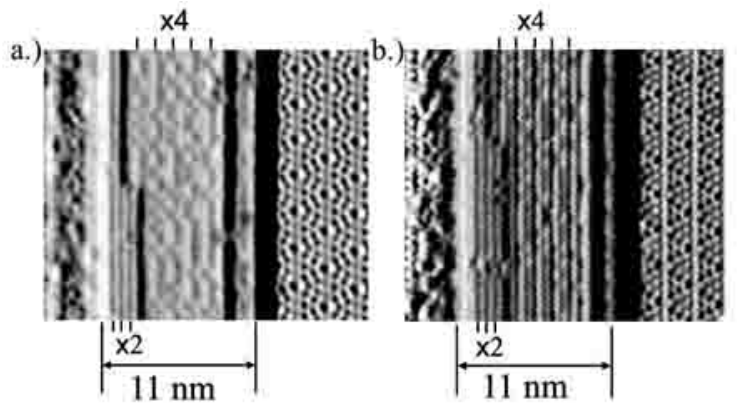

Fig. 4 Close-up of the surface reconstruction on top of a nanowire at two sample bias voltages $(+2 \mathrm{~V}$ on the left, $-2 \mathrm{~V}$ on the right). Two types of chain structures are observed with a chain spacing of 2 and 4 lattice constants $(\times 2$ and $\times 4)$.

growth of atomically-precise $\mathrm{Gd}$ disilicide nanowires on $\mathrm{Si}(111)$. The typical width is $10 \mathrm{~nm}$ and the length can exceed a $\mu \mathrm{m}$. The growth mechanism is driven by lattice match between the a-axis of the silicide and the [ $\left.\begin{array}{lll}1 & \overline{1} & 0\end{array}\right]$ direction along the $\mathrm{Si}$ surface, as observed for a number of rare earth disilicides on $\mathrm{Si}(100)$. However, on $\mathrm{Si}(111)$ the nanowires grow only at step edges. That allows some degree of control over their position, which is the first step towards self-assembled interconnects between nano-devices at a surface. The next step will be the search for a nucleation mechanism that would make it possible to position the beginning or end of a nanowire along a step.

Acknowledgment: This work was supported by the NSF (DMR-9815416 and DMR-0079983) and by the DOE under Contract No. DE-FG0201ER45917.

\section{References}

1. M. Sastry, A. Kumar, S. Datar, C.V. Dharmadhikari, and K.N. Ganesh, Appl. Phys. Lett. 78, 2943 (2001).

2. J. Tersoff and R.M. Tromp, Phys. Rev. Lett. 70, 2782 (1993).
3. P. Kluth, Q.T. Zhao, S. Winnerl, S. Lenk, and S. Mantl, Appl. Phys. Lett. 79, 824 (2001).

4. S.H. Brongersma, M.R. Castell, D.D. Perovic, and M. Zinke-Allmang, Phys. Rev. Lett. 80, 3795 (1998).

5. V. Dorna, M. Kneppe, K. Reshöft, and U. Köhler, Surf. Sci. 454-456, 802 (2000).

6. T.H. McDaniels, J.A. Venables, and P.A. Bennett Phys. Rev. Let 87, 1 (2001).

7. For a review of rare earth overlayers on silicon see F. P. Netzer, J. Phys. Condens. Matter 7, 991 (1995).

8. C. Preinesberger, S. Vandré, T. Kalka and M. Dähne-Prietsch, J. Phys. D 31, L43 (1998).

9. Y. Chen, D.A.A. Ohlberg, G. Medeiros-Ribeiro, Y.A. Chang, and R.S. Williams, Appl. Phys. A 75 , 353 (2002).

10. J. Nogami, B.Z. Liu, M.V. Katkov, C. Ohbichi, and N.O. Birge, Phys. Rev. B 63, 23305 (2001).

11. L. Fitting, M.C. Zeman, W. Yang, R.J Nemanich, A.P.S. March Meeting Bulletin, B22 6. (2002)

12. J.A. Knapp and S.T. Picraux, Appl. Phys. Lett. 48, 466 (1986).

13. S. Auffret, J. Pierre, B. Lambert-Andron, R. Madar, E. Houssay, D. Schmitt, and E. Siaud, Physica B 173, 265 (1991).

14. S. Vandré, T. Kalka, C. Preinesberger, and M. Dähne-Prietsch, Phys. Rev. Lett. 82, 1927 (1999).

15. C. P. Collier, G. Mattersteig, E.W. Wong, Y. Luo, K. Beverly, J. Sampaio, F. Raymo, J.F. Stoddart, and J.R. Heath, Science Science 289, 1172 (2000).

16. T. Rueckes, K. Kim, E. Joselevich, G. Y. Tseng, C.L. Cheung, and C.M. Lieber, Science 289, 94 (2000).

17. J.-L. Lin, D.Y. Petrovykh, J. Viernow, F.K. Men, D.J. Seo, and F.J. Himpsel, J. Appl. Phys. 84, 255 (1998).

18. The diffusion of $\mathrm{Gd}$ into $\mathrm{Si}$ above $700^{\circ} \mathrm{C}$ is evidenced by a gradual transformation of the $\operatorname{Si}(111) 5 \times 2$-Gd surface into clean $\operatorname{Si}(111) 7 \times 7$. The reverse effect is observed as well, i.e., segregation of Gd from the bulk to form patches of $\operatorname{Si}(111) 5 \times 2-\mathrm{Gd}$. This happens after depositing several monolayers of $\mathrm{Gd}$ and diffusing them into the bulk at $1250^{\circ} \mathrm{C}$.

19. A. Kirakosian, J. L. McChesney, R. Bennewitz, J. N. Crain, J.-L. Lin, and F. J. Himpsel, Surf. Sci. 498, L109 (2002). 